\title{
Attitude, knowledge and overall perception towards working under magnification among undergraduate and postgraduate students of a dental institution in Andhra Pradesh-A questionnaire based study
}

\author{
Kausalya Devi Panda ${ }^{1 *}$, Gautami. S. Penmetsa ${ }^{2}$, Radha Rani $K^{3}$, Jaswitha $V^{4}$ \\ 1,3,4Post Graduate Student, ${ }^{2} \mathrm{HOD}$, Dept. of Periodontics and Implantology, Vishnu Dental College, Bhimavaram, Andhra Pradesh, India
}

\author{
*Corresponding Author: Kausalya Devi Panda
}

Email: kausineeru@gmail.com

\begin{abstract}
Introduction: Dentistry often referred to an art is that profession that requires mastery and techniques along with profound scientific knowledge. It has taken its place from macro dentistry to micro dentistry with tissue precision and less trauma. Procedures under magnification devices require a steep learning curve which make the attitude of students negative towards using magnification. After a constant training period it improves the ergonomic benefits of the students.

Results: Among 179 undergraduate and postgraduate students a questionnaire was given with different sets of questions where $82.5 \%$ of students felt that it is comfortable working under magnification and around $92.4 \%$ of students don't want to revert back to conventional procedure and they felt there is improved ergonomic benefit while working under magnification.

Conclusion: The present study indicates that training under magnification should be started under early practice of every student. Procedures under magnification improves the ergonomic benefit, visual acuity and duration of treatment while compared to conventional procedures.
\end{abstract}

Keywords: Magnification devices, Ergonomic benefit, Visual acuity, Magnification dentistry.

\section{Introduction}

Dentistry is an art and science that requires good hand, eye, and mind co-ordination along with the posture of clinician which makes up the most important criteria. Ergonomics is one such aspect of dentistry that focuses on a co-ordination of all the above said factors thereby preventing musculoskeletal disorders. It is that scientific discipline concerned with the understanding of interactions among humans and other elements of system applying to the theory, principles, data and methods designed in order to optimize human wellbeing and overall system performances. ${ }^{1} \mathrm{~A}$ proper successful treatment is dependent on a proper magnification and varying usage of magnification enhanced with proper visualization which not only reduces the working distance but also helps in maintaining correct posture, thereby improving the motor skills of operator. ${ }^{2}$

It is interesting to state that the literature has evidenced the prevalence of Musculoskeletal disorders (MSD) around $82.9 \%$ in dentistry thereby succumbing the dentist to neck pain, discomfort from neck, shoulders lower back pain and head. ${ }^{3}$ This MSD being the utmost reason for early retirement of dentist, each and every dentist needs to adapt and guarantee good working postures enhanced with improved magnification during various clinical protocols and approaches for reduction in ergonomic problems that include awareness of postural techniques, postural and positioning of patients and utilization of magnification systems. ${ }^{1,3}$ The magnification system ranges from Loupes to Surgical Operating Microscope (SOM) and a good choice of magnification device along with usage of micro surgical instruments helps in improvement of ergonomics. ${ }^{4}$ Loupes are widely used in dentistry which is two monocular microscopes lens positioned side by side and angle to focus on single object. Various types of loupes range from simple loupes to compound loupes to Galilean loupes to prism loupes. These loupes provide better field of vision, better treatment outcome inspite of its steep learning curve and expensiveness. ${ }^{5}$ On the other hand SOM is definitely a better substitute because of its varying advantages of improved magnification and better ergonomics. However, the limitations of its use lies in its steep learning curve and its affordability thereby restricting its use.

As in the very recent years literature emphasizes the prevalence of MSD in dentists, dental hygienists and dental students thereby precluding them to early onset of MSD's. It is the responsibility of the clinician to educate the dental students and dental hygienists on the various positional postural strategies consists of avoiding constant posture, positioning patient in proper height, rotating shoulder backwards and usage of back rest. ${ }^{6}$ Taking all the above said factors into consideration, loupes provide better field of vision and better treatment outcome inspite of its steep learning curve and expensiveness. Keeping this in mind we wanted to emphasize the importance of magnification thereby reducing MSD and emphasizing the overall benefits for both patients and clinician as well. As we in our institution focused on usage of magnification devices at a very early stage, the main aim of us in conducting this study was to assess the attitude, knowledge and overall perception among undergraduate and postgraduate students of Vishnu dental college.

\section{Materials and Methods}

This questionnaire analysis was conducted from May 2019 to June 2019 which was given to Interns and Postgraduate students of Vishnu Dental College Bhimavaram who were well aware and subjected to conventional practice of dentistry and were subjected to the usage of magnification device after some point of time in clinical experience in order to assess 
their attitude, perception, and overall assessment before and after usage of loupes in their clinical experience.

A questionnaire comprising of 20 questions was prepared out of which 8 questions were based on the attitude, 6 questions were based on perception during treatment procedure and rest 6 questions were based upon their overall assessment of the clinician while working with and without magnification device. Few of the questions were included from previously published study which were modified and included in our questionnaire. The questionnaire was validated and then incorporated in our study.

\section{Statistical analysis}

The data were collected, entered into MS Excel spreadsheet and analyzed through the statistical package for the social sciences package v. 20 software (SPSS Inc. Chicago, IL, USA). Simple descriptive statistics were used to define characteristics of the variables using numbers and percentages for categorical variables. To establish relationships between categorical variables, $\mathrm{Chi}^{-}$square test was used, and statistical significance was recognized when $\mathrm{P}$ $<0.001$.

\section{Results}

The questionnaire employed in our study constituted of 20 questions which we have divided into three different sets. The first set constitutes 8 questions to evaluate the attitude of the practitioners while using magnification devices in their treatment procedures. Second set of 6 questions were framed on their perception of using the magnification devices during their working time. The rest of the questions were framed on the overall assessment of the practitioners during the usage of magnification devices throughout the treatment.

Among 171 practitioners 90 were postgraduates from all the departments who were not experienced working under magnification in their under graduation and the remaining 81 were interns who started their journey under magnification in their early clinical practices.

When the question of cost effectiveness is posed around $77.8 \%$ have come out with an opinion that usage of surgical loupes was expensive due to which around $82.4 \%$ of students felt its use as a disadvantage in their clinical practice. Our next question was focused on the comfort of wearing the loupes where we had 21 patients using glasses for their vision. Among these 20 students almost $65 \%$ of them complained that wearing a magnifying loupe along with their glasses caused discomfort and eye strain. As it was a training period for all the clinicians, $74.5 \%$ of them who were subjected to usage of magnification has started it as they were forced by their teachers to practice their treatment procedures only under magnification. $89.7 \%$ of students felt they need training classes for 3-6 months to get experienced into using magnification devices. It was a pleasant finding from our side to come out with a result that, around $70 \%$ of students felt treatment under magnification was more comfortable than conventional procedure after getting accustomed to it.

In our second set of questions on their perception of using magnification while treatment procedures were performed, $95 \%$ of the students experienced change in the duration of treatment under magnification which was faster than under conventional procedure. A satisfactory result was achieved regarding the treatment outcome and ergonomic benefit of the clinician where around $87.9 \%$ agreed that ergonomic benefit and quality of treatment had improved under magnification compared to that of a conventional procedure. We have noted that for a question on satisfaction level of the student while working under magnification, $85 \%$ of the students came out with an opinion that after good training period they are more comfortable to work under loupes.

In the last set of questions which are posed on overall perception of the students regarding a question on type of procedure they are comfortable under magnification among 81 interns around $65(80 \%)$ of interns shared their opinion that restorations and scalings are more comfortable procedures to carry out under loupes. Among 90 postgraduates, 18 postgraduates from the department of endodontics came out with a $100 \%$ result that endodontic procedures gave better treatment outcome while working under loupes when compared to conventional procedure. There were total of 15 postgraduates from the department of periodontics wherein among them $95 \%$ of students shared their opinions that both non- surgical and surgical procedures were more comfortable along with good treatment quality under magnification than under conventional procedures. $6.4 \%$ of students also felt that surgical extractions also had a good result under magnification. Among 18 postgraduates from the department of Prosthodontics, 65\% of students were comfortable using loupes during tooth preparation and crown cementation. Out of the 18 postgraduates from Orthodontics department only $43.1 \%$ of students were comfortable using magnification during banding and bonding procedures. Among 18 postgraduates from pedodontics $94 \%$ were comfortable working under loupes during scalings, restoration, pulpotomy and pulpectomy procedures. A question posed on benefits of the patients while getting their treatment under magnifications, $60 \%$ of students felt that patients are not that comfortable to get their treatment done under magnification. We have also made an attempt to evaluate the difference while working under conventional procedures and loupes. In this set of questions we got a result of $85 \%$ where the students used to suffer with back pain and neck pain which was reduced among $94 \%$ of students. On the other had around $53.9 \%$ have complained of eye strain and headache while working under magnification.

As a whole, on overall assessment we are pleasant to note that for a question posed on their continuation of treatment under magnification around $92.6 \%$ of students shared their opinion saying they don't want to revert back to the conventional procedure and they are comfortable working under magnification. 
Graph 1: Showing the attitude of the students towards using magnification devices.

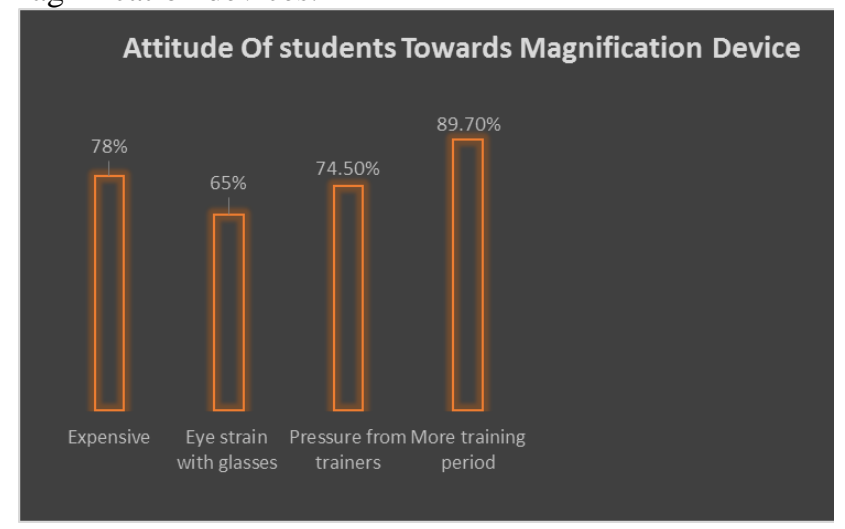

Graph 2: Showing the ergonomic benefit of the student before and after usage of the magnification device.

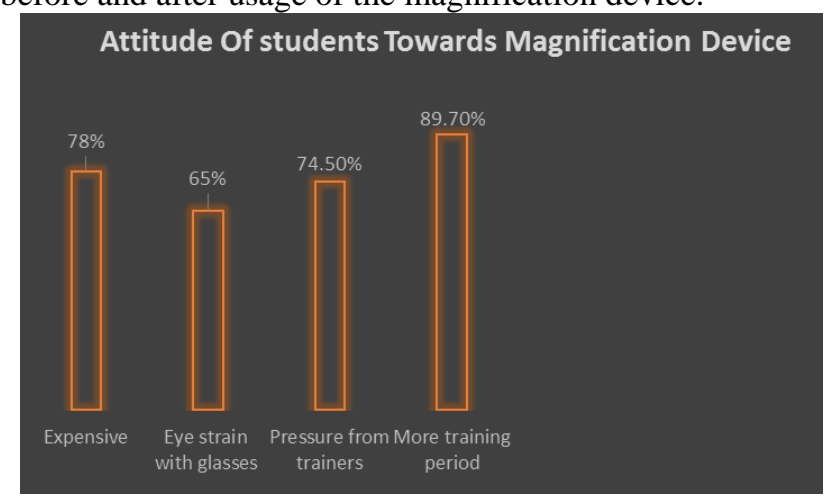

Graph 3: Showing the comfort level of the student towards using magnification device.

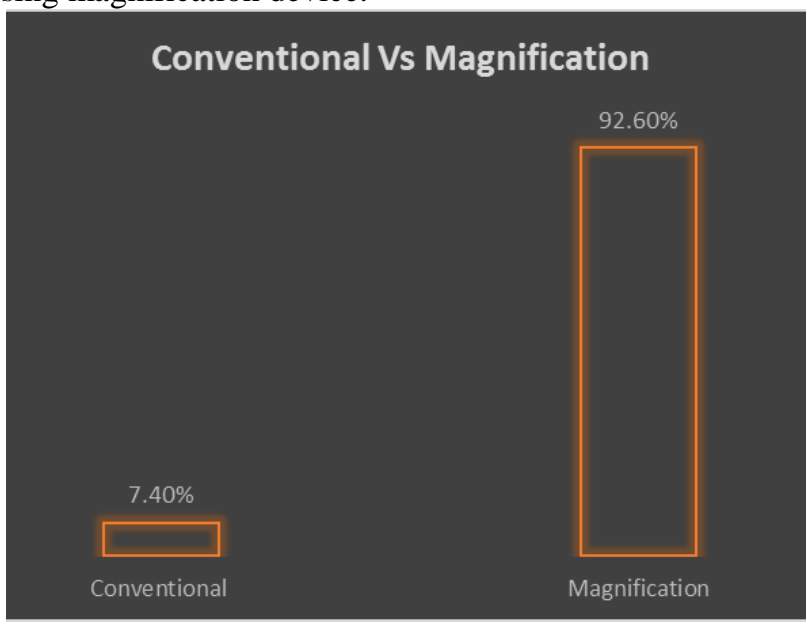

\section{Discussion}

In the evolving field of dentistry, where it has initiated its journey with naked eyes and reading glasses magnification took a lead role in every sphere of this ever growing science. A better vision gives better manual dexterity with an improved treatment quality for the innumerable procedures executed in daily dentistry. ${ }^{7}$ Surgical approaches especially on soft tissues usually require precise handling which is enhanced using magnification. In this context it is appropriate to state that today's dentist is left with numerous magnification devices like $2.5 \mathrm{X}$ loupes, loupes with higher magnifications along with headlight and vast varieties of surgical operating microscopes. The rationale being the usage of magnification devices was to give a quality care treatment with less trauma, less pain and more comfort to the clinician along with attaining holistic results which can be attributed to its precise approach. This enhances the motor skills of the clinician along with ergonomics while performing treatment procedures. $^{8}$

As the goal of today's dentistry has heightened to provide quality care treatment. Magnification has played a better role in all the specialties from giving a better diagnosis to better treatment outcome. Diagnosis of small ulcers, oral lesions in the oral cavity, analyzing the radiographs, treating a carious teeth i.e. from a simple restoration to a root canal procedure, periodontal procedures both non-surgical and surgical, crown preparations with a fine finish margin, surgical extractions and orthodontic bracket placements got better treatment outcomes under magnification than under conventional procedure. ${ }^{4}$

As the above considerations are the prerequisites of providing better treatment outcomes for the patients in our study, we aimed to analyze the attitude, perception and overall assessment of every clinician who are exposed to both the conventional procedures as well magnification procedures. Hence an attempt was made by us to know the role of magnification devices in the clinical practices of budding dentists who are in their early usage of magnification. Inspite of its advantages their use is usually limited in the field of dentistry which may be because of varied attitudes of the individuals.

All our subjects being students, we found their attitude towards usage of magnification in their early practice to be negative which may be because of expense incurred in procuring the device and steep learning curve when compared to conventional procedures. However many students started their journey of using magnification devices only because of the external pressures from their trainers. Around $95 \%$ students have stated that there was change in the duration of the treatment while working under magnification when compared to conventional procedures which was in accordance with the study done by Narula at.al which states that students who used magnification loupes have done more number of cases in less time when compared to the students who treated conventionally. ${ }^{9}$

Inspite of many advantages magnification has got its limitations because of its steep learning curve. In our study around $89.7 \%$ of students felt that they need training classes to work more comfortably under magnification among whom most of them were interns. Compared to interns postgraduates felt that they were more comfortable working under magnification devices as they attended courses on magnification. This was in accordance with the study done by Alhazzazi et al where they have found that undergraduates were more restricted to use magnification devices where the postgraduates were more comfortable to work under 
magnification..$^{10}$ This is evident of the fact that it is as earlier the exposure better will be the enhancement of clinical skills working under magnification devices.

On the other hand musculoskeletal health of the dentists has been subjected as a matter of debate worldwide. It is interesting to note that around $85 \%$ of students complained of back pain, neck pain and shoulder pain which was reduced among $94 \%$ of students after working under magnification. This results were in accordance with a report which have stated that one out of 10 dentists have poor general health and every three out of 10 dentists have poor physical state which was reduced after increasing awareness towards ergonomics during dental practice. It is always better to employ the usage of magnification device in the early training period of dental students which can significantly improve their posture. ${ }^{10,11}$

It was also analyzed in our study that only $53.9 \%$ of students have experienced eye strain after working under magnification which was almost a less number. This was in accordance to the report given by Christensen who stated that after working under magnification eyes require time to readjust to normal vision. ${ }^{12}$

There was no literature stating the comfort of the patient while working under magnification. Clinicians in our study even reported that $50 \%$ patient was not that comfortable while working under magnification owing to involuntary focusing of light emitted from the loupes on their faces. Our overall assessment has analyzed that majority of the operators (92.6\%) were comfortable working under magnification if they are employed into the clinical practice as early as possible in their training periods and they don't want to revert back to conventional procedures. We are privileged to add a note that our institution has already took its step in advocating micro dentistry in every clinician's day to day practice which is in agreement with many dental schools worldwide which are now actively encouraging all the undergraduates and postgraduate students towards use of dental magnification.

\section{Conclusion}

The advancement in science and technological progresses facilitated the evolution of different treatment procedures in the field of dentistry. One such magnified invention is the concept of microdentistry by using different magnification devices where dentistry took a huge turn. Every advancement may end up with few limitations likewise here magnification may have few limitations such as steep learning curve and expensive equipments but it has made the job of a dental surgeon more precise with excellent soft tissue management, perfect tissue approximation with added benefits of improved ergonomics to the operator and better esthetic outcome to the patient along with less post-operative pain. Magnification is always a better treatment philosophy needed to be followed by every dental surgeon whose clinical horizons will tend to improve with perpetual practice in their day to day life.

\section{Source of funding}

None.

\section{Conflict of interest}

None.

\section{References}

1. Gupta A, Bhat M, Mohammed T, Bansal N, Gupta G. Ergonomics in Dentistry. Int J Clin Pediatr Dent 2014;7(1):304.

2. Carlos M. Microdentistry, concept, methods and clinical incorporation. Int J Microdent 2010;2:56-63.

3. H.S. Bedi. Evaluation of MSD in Dentists and Application of DMAIC Technique to Improve the Ergonomics at Dental Clinics. J Clin Diagn Res 2015;9(6).

4. Hegde R, Hegde V. Magnification-enhanced contemporary dentistry: Getting started. J Interdiscip Dent 2016;6:91-100.

5. Tibbetts LS, Shanelec DA. Principles and Practice of Periodontal Microsurgery. Int J Microdent 2009;1:1-23.

6. Evangelos C A, Ioanna C S, Fotini C. Prevalence of musculoskeletal disorders in dentists. BMC Musculoskelet Disord 2004;5:16.

7. Jun F L, Tuti N M, Safura A B. Magnification in endodontics: A review of its application and acceptance among dental practitioners. Eur J Dent 2018;12:610-6.

8. Mallikarjun SA, Devi PR, Naik AR, Tiwari S. Magnification in dental practice: How useful is it?. J Health Res Rev 2015;2:39-44.

9. Narula K, Kundabala M, Shetty N, Shenoy R. Evaluation of tooth preparations for Class II cavities using magnification loupes among dental interns and final year BDS students in preclinical laboratory. J Conserv Dent 2015;18:284-7.

10. Alhazzazi TY, Alzebiani NA, Alotaibi SK, Bogari DF, Bakalka GT, Hazzazi LW et al. Awareness and attitude toward using dental magnification among dental students and residents at King Abdulaziz University, Faculty of Dentistry. BMC Oral Health 2016;17:21.

11. Aghilinejad M, Kabir-Mokamelkhah E, Talebi A, Soleimani $\mathrm{R}$, Dehghan N. The effect of magnification lenses on reducing musculoskeletal discomfort among dentists. Med J Islam Repub Iran 2016;30:473.

12. Christensen GJ. Magnification in dentistry: Useful tool or another gimmick? J Am Dent Assoc 2003;134:1647-50.

How to cite this article: Kausalya Devi Panda, Gautami. S. Penmetsa, Radha Rani K, Jaswitha V. Attitude, knowledge and overall perception towards working under magnification among undergraduate and postgraduate students of a dental institution in Andhra Pradesh- A questionnaire based study. Int J Periodontol Implantol 2019;4(3):88-91. 